\title{
Magneto-optical characteristics of magnetic nanowire arrays in anodic aluminum oxide templates
}

\author{
Yong Peng, T.-H. Shen, ${ }^{a)}$ Brian Ashworth, Xue-Gen Zhao, Chester A. Faunce, \\ and Yan-Wei Liu \\ Joule Physics Laboratory, Institute for Materials Research, Salford University, Salford, M5 4WT, United Kingdom
}

(Received 27 February 2003; accepted 19 May 2003)

\begin{abstract}
Nanocomposite films consisting of regularly ordered iron nanowires embedded in anodic aluminum oxide templates have been fabricated and their magneto-optical properties studied by determining the four Stokes parameters of the transmitted laser beam $(\lambda=670 \mathrm{~nm})$, originally linearly polarized and at normal incidence to the film surfaces. The results of the nanowire arrays are found to be considerably different from that of bulk iron. While an increase in diameter of the nanowire leads to a substantial increase in the values of the Faraday rotation angles per unit length at a fixed value of the magnetic fields, they are substantially less than that of bulk iron, indicating that the effective media theory may not be directly applicable. (C) 2003 American Institute of Physics.
\end{abstract}

[DOI: $10.1063 / 1.1590427]$

Although arrays of dots or nanorods ${ }^{1}$ made by lithographic techniques have been investigated by magnetooptical Kerr effect (MOKE), ${ }^{2,3}$ and for nanowire arrays, the absorption spectra by using UV-Vis spectroscopy, ${ }^{4,5}$ the study of magneto-optical related properties of nanowires based on porous anodic aluminum oxide (AAO) templates has been surprisingly sparse. ${ }^{6,7}$ In this letter, we report on a detailed experimental study of the linear magneto-optical properties of iron nanowire arrays embedded in AAO templates measured at normal incidence to the composite films.

Our optical setup is as follows. A light beam from the source (a diode laser, Laser 2000, $1.85 \mathrm{eV}, 3 \mathrm{~mW}$ ) passed through a polarizer, the center hole of the first pole piece of a magnet, a sample, the second pole piece, a photo-elastic modulator (PEM, Hinds Instruments, $f=42 \mathrm{kHz}$ ), an analyzer, and finally to a Si photodiode detector. The polarizer and analyzer were of an extinction ratio better than 2 $\times 10^{-6}$. To ensure no reflections from the internal walls of the narrow center holes in the soft iron cores, two small apertures were placed, one at the entrance to the first coil and the other at the exit from the second. A Hall probe was placed very close to the sample to simultaneously monitor the magnetic fields. The signals from the photodiode were measured with an EG\&G 5209 lock-in amplifier and the dc component of the photodiode signal was measured with a high-precision Keithley 6517 electrometer. The data acquisition was automated using a personal computer. Following $\mathrm{Kemp}^{8}$ the Stokes parameters ${ }^{9}$ were then determined from the measured dc signal, as well as the first- and secondharmonic signals. For an absolute measurement of the parameters, the setup was "calibrated" using a quarter-wave plate in the place of the sample.

The composite films were prepared by anodizing thin sheet of high purity (99.99\%) aluminum electrochemically, followed by electrochemical deposition of iron wires in an appropriate aqueous solution, as reported in an earlier work. ${ }^{10}$ Details of our sample preparation will be published

\footnotetext{
a) Author to whom correspondence should be addressed; electronic mail: t.shen@salford.ac.uk
}

elsewhere. The films had a degree of transparency to visible light. The AAO templates were typically about $6 \mu \mathrm{m}$ thick, as determined from the interference patterns in the optical absorption spectra. A range of samples with different wire diameters and lengths were prepared and investigated. The structures of the composite films were characterized by scanning electron microscopy (SEM). The total cross-sectional areas of the wires were found to be about $30 \%$ to $50 \%$ of the composite film surfaces, for a range of wire diameters, which were controlled by the electrochemical conditions during preparation. The nanowires in the templates were hexagonally close packed and ordered to a high degree. Very roughly, the diameter of the wires and the space between them were of similar sizes. To determine the actual diameter and the length of the nanowires, the AAO templates were dissolved and the individual wires measured by transmission electron microscopy (TEM). An average value of length and diameter was obtained for a number of measurements.

Figure 1 shows typical electron micrographs of the AAO templates and the nanowire arrays. Figure 1(a) is a SEM image of a gold-coated AAO template having 55-nmdiameter pores, showing a high degree of order and uniformity. The space between the nearest-neighbor pores is about $30 \mathrm{~nm}$, slightly smaller than the pore diameter. A TEM cross-
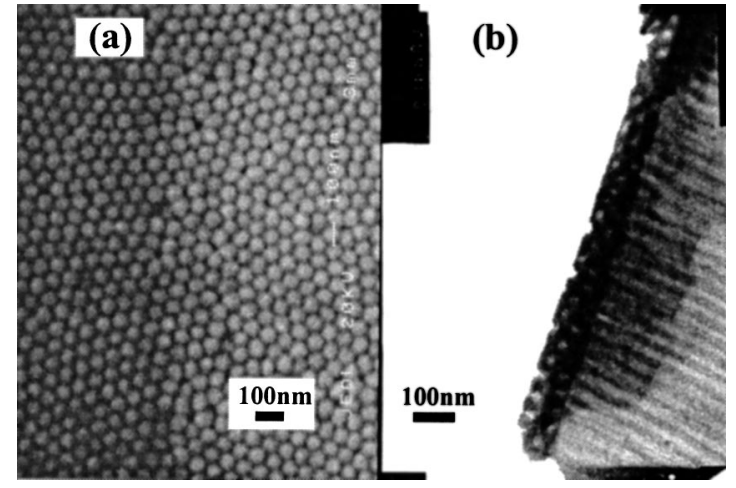

FIG. 1. Electron micrographs of AAO templates and Fe nanowire arrays: (a) SEM image of an AAO template with 55-nm pores; and (b) TEM crosssectional image of $\mathrm{Fe}$ nanowires (15-nm diameter) embedded in an AAO template. 


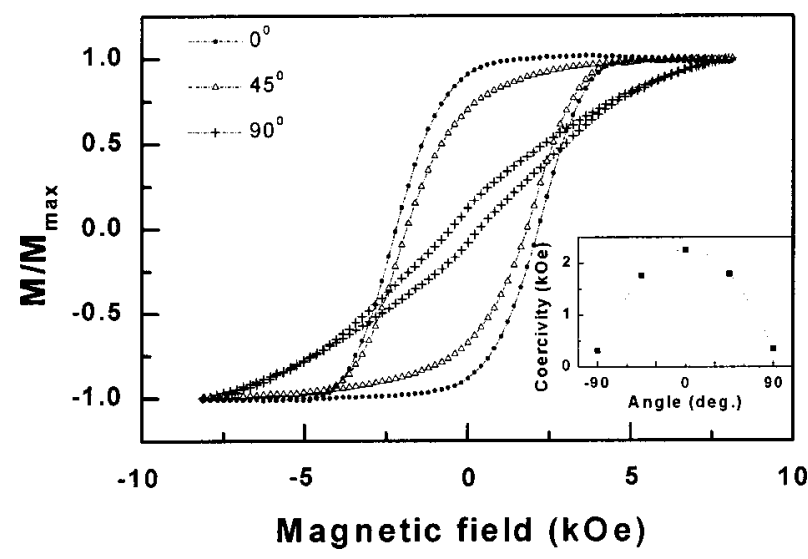

FIG. 2. VSM results of magnetization for a 7-nm-diameter Fe nanowire array, measured at different values of the angle between the magnetic field and the surface norm of the nanocomposite film. Inset shows the corresponding coercivity.

sectional image of an $\mathrm{Fe}$ nanowire array embedded in an AAO template is displayed in Fig. 1(b), which shows that the 15-nm-diameter Fe nanowires filled the nanochannels uniformly as well as showing that the wires were parallel to each other and perpendicular to the template surface. In addition, one can see that the closest distance between two pores is about $20 \mathrm{~nm}$, slightly larger than relevant pore diameter. The selective area electron diffraction pattern, of nanowire bundles, indicates that the bulk bcc structure is preserved.

Figure 2 shows the results of the magnetization measurements for a "thin" nanowire array (referred to as sample A, wire diameter $7 \mathrm{~nm}$, wire length $2.13 \mu \mathrm{m}$ ). The hysteresis loops were obtained at different angles between the magnetic field direction and the surface normal of the nanocomposite films using a vibration sample magnetometer (VSM). The angular dependence of the magnetization loops illustrated that the easy axis of the nanocomposite film is along the surface normal, that is, along the length of the nanowires. The inset in Fig. 2 shows the values of the coercivity as a function of the angle between the applied fields and the surface normal of the film. A coercivity of about $2.5 \mathrm{kOe}$ is found along the easy axis and is consistent with earlier work on nanowires. ${ }^{10}$

In Figs. 3(a) to 3(d), as an example, the Stokes parameters of the same sample, normalized by the intensity of the polarized component, are presented. The first two curves are the normalized first [Fig. 3(a)] and second [Fig. 3(b)] Stokes parameters. The curves in Figs. 3(c) and 3(d), $s_{3}^{\prime}\left(0^{\circ}\right)$ and $s_{3}^{\prime}\left(45^{\circ}\right)$, respectively, are the normalized third Stokes parameter measured at two different PEM positions, and the resulting curves are found to be identical..$^{9}$ Both $s_{3}^{\prime}\left(0^{\circ}\right)$ and $s_{3}^{\prime}\left(45^{\circ}\right)$ are measured as a check on the quality of the results. The measured Stokes parameters showed good signal-tonoise characteristics. The ratio of the axes $a$ and $b$ of the ellipse, $\tan (\chi)$, for sample A is shown in Fig. 3(e). A very good agreement may be seen between the hysteresis loop in Fig. 3(c) and the loop obtained from the VSM shown in Fig. 2. The data illustrate that $\tan (\chi)$ is a good measure of the magnetization behavior of the nanocomposite films. It may also be noted that the ratio of the axes of ellipse is about $1 \%$, indicating that the originally linearly polarized light is virtu-
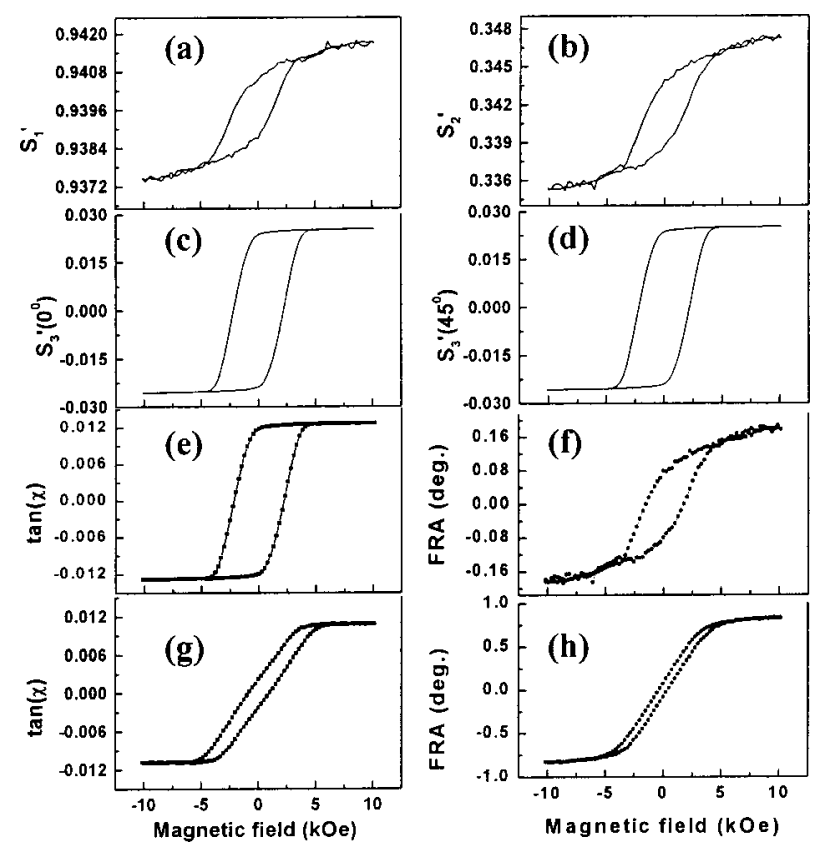

FIG. 3. Magneto-optical results: (a)-(d) normalized Stokes parameters for a 7-nm-diameter Fe nanowire array; (e) the ratio of ellipse $\tan (\chi)$ and (f) FRA as a function of magnetic fields for the same sample; and $(\mathrm{g}) \tan (\chi)$ and FRA for a 144-nm-diameter Fe nanowire array.

ally unchanged after exiting the sample. The magnetic circular dichroism (MCD) is thought to be the origin of the magnetic dependence in $\tan (\chi)$. A direct measurement of the MCD with alternating left-hand and right-hand circular polarized light confirms the observed value.

The Faraday rotation angle (FRA) of sample A is plotted in Fig. 3(f), showing a more complex field dependence. A sloping "background" appears to have been superimposed on the hysteresis loop. This background is seen more prominently in Fig. 3(f) as the Faraday rotation angles are relatively small compared to that of a thicker nanowire array [cf., Fig. 3(h)]. Although a detailed quantification of the background for all samples has yet to be carried out, the magnetooptical effect of the AAO templates without the nanowires has been examined. The paramagnetic Faraday rotation angles of the templates are found to be about $1 / 5$ the values of the background in the nanowire arrays, and therefore could not directly account for the effect noticed in Fig. 3(f). At present, the origins of the background are not fully understood.

Figures 3(g) and 3(h) show the magneto-optical results of a thicker sample (referred to as sample B, wire diameter $144 \mathrm{~nm}$, wire length $1.57 \mu \mathrm{m})$, and the $\tan (\chi)$ data are plotted in Fig. $3(\mathrm{~g})$. It can be seen that as the wire diameter is increased, the coercivity decreases. At saturation magnetization, the values of $\tan (\chi)$ also are slightly smaller compared with that of sample A. This is in line with a shorter length of the nanowires for sample B. Despite the shorter length, the Faraday rotation angles at maximum fields are much larger than that of sample A. As mentioned earlier, the sloping background is also present in the Faraday rotation data, although it appears less prominent in the diagram.

We shall now examine the magneto-optical characteristics of the nanowire arrays as a function of the wire lengths and diameters. In Fig. 4(a), the FRAs at the maximum field to AIP license or copyright, see http://ojps.aip.org/aplo/aplcr.jsp 

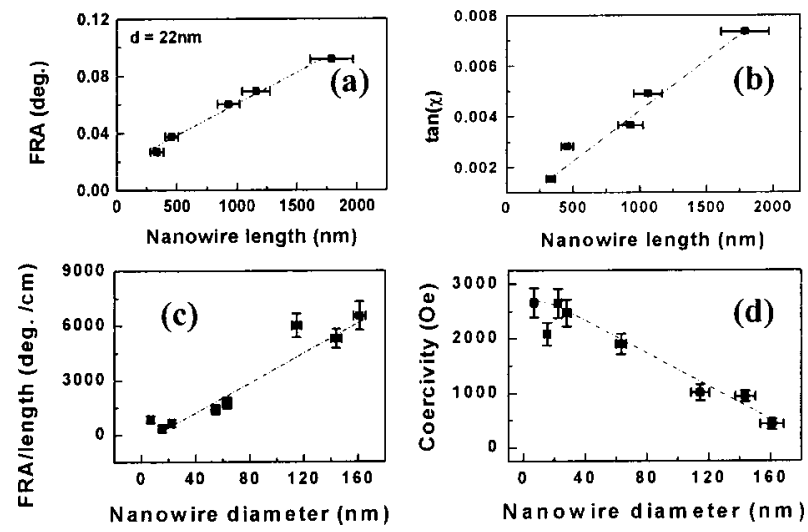

FIG. 4. (a) FRA versus nanowire length for 22-nm-diameter wire arrays; (b) corresponding $\tan (\chi)$ as a function of nanowire length; (c) FRA/length as a function of nanowire diameter; and (d) coercivity as a function of nanowire diameter as obtained from the $\tan (\chi)$ data. The dotted lines in the diagrams are a guide to the eyes only.

of $10 \mathrm{kOe}$ are displayed as a function of the lengths of the nanowires for the composite films with a wire diameter of 22 $\mathrm{nm}$. (In the absence of a clear mechanism, the sloping background is not subtracted, which would have given rise to a correction of less than $10 \%$ of the lower values of the rotation angles. This should not affect our discussion on the overall trends.) As can be seen in the diagram, the rotation angles are essentially proportional to the length of the wire, which is consistent with the Faraday effect. If the rotation angle is expressed in terms of the product of a Verdet constant $V$, the external field $H$, and the length of the wire $l$, we obtain a value $V=7.2 \times 10^{-2} \operatorname{deg} \mathrm{Oe}^{-1} \mathrm{~cm}^{-1}$ at the field of the onset of the saturation magnetization, which is nearly three orders of magnitude smaller than that of bulk iron ( $V$ $\left.=3.8 \times 10^{1} \mathrm{deg} \mathrm{Oe}^{-1} \mathrm{~cm}^{-1}\right){ }^{11}$ The value is also about 10 times larger than that of iron nanoparticles in poly(methylmethacrylate $)\left(V=6.0 \times 10^{-3} \mathrm{deg} \mathrm{Oe}^{-1} \mathrm{~cm}^{-1}\right.$, at $\lambda=630$ $\mathrm{nm}) .{ }^{12}$ Compared with a typical paramagnetic material of Schott glass SF-57 $\left(V=2.264 \times 10^{-4} \mathrm{deg} \mathrm{Oe} \mathrm{cm}^{-1}\right.$, at $\lambda=1300 \mathrm{~nm}),{ }^{13}$ the Verdet constant for the 22 -nm-diameter nanowire arrays is only about two orders of magnitude larger than that of Schott glass. In Fig. 4(b), the ratio of the axes, $\tan (\chi)$, for the 22-nm wire arrays also increases with the increasing lengths of the wires. This could be explained in terms of a stronger MCD effect with increasing length, because of the increase in absorption in longer wires.

In Fig. 4(c), the values of FRA/length at the maximum fields are plotted as a function of nanowire diameters. It can be seen that the FRA/length increases as the wire diameter increases. The largest diameter sample $(160 \mathrm{~nm})$ shows a FRA/length value of $6.5 \times 10^{3} \mathrm{deg} \mathrm{cm}^{-1}$, while the smallest one $(7 \mathrm{~nm})$ has a value of only $8.5 \times 10^{2} \mathrm{deg} \mathrm{cm}{ }^{-1}$. The bulk $\mathrm{Fe}$ is known $^{11}$ to have a FRA/length of about 3.8 $\times 10^{5} \mathrm{deg} \mathrm{cm}{ }^{-1}$, which is between two to three orders of magnitude larger than that of the nanowire arrays. We note that the percentage of iron wire cross sections occupying the surface area of the films is between 30 and 50, and the absolute value of the saturation magnetization $M_{s}$ per nanowire volume is estimated to be $5 \times 10^{5} \mathrm{~A} \mathrm{~m}^{-1}$ for $15-\mathrm{nm}$ diameter wires. Based on Almawlawi et al. ${ }^{14}$ we estimate a value about $1.9 \times 10^{6} \mathrm{~A} \mathrm{~m}^{-1}$ for their sample (the first sample of Sec. 2 of Table III ${ }^{14}$ ), whose experimental condi- tions were close to our 15-nm-diameter sample. In either case, we note that $M_{s}$ per nanowire volume is of the same order of magnitude as that of bulk iron $\left(1.71 \times 10^{6} \mathrm{~A} \mathrm{~m}^{-1}\right.$ at room temperature). Despite the fact that the structures of the nanocomposite films are substantially smaller than the wavelength of the light, the large differences in the values of FRA/length appear to suggest that the effective media theory ${ }^{15}$ may not be directly applicable.

Figure 4(d) shows the coercivity in relationship with the nanowire diameters, as determined from hysteresis loops of $\tan (\chi)$, for example, the curves in Figs. 3(e) and 3(g). Experimentally, it is not a trivial task to make different diameter nanowire arrays of identical wire lengths. However for the set of 22-nm-diameter Fe nanowire arrays of different wire length (ranging from 0.7 to $2.5 \mu \mathrm{m}$ ), we have found a slightly changed value for the coercivity, within a change of a few percentage points. This suggests that the results in Fig. 4(d) would be good enough to indicate the general trend that is a sharp reduction of the coercivity as the diameter of the nanowires is increased.

Additionally, from the results of a complete set of Stokes parameters, it is also possible to show that the degree of polarization ${ }^{9}$ begins to decrease for nanocomposite films with wire diameter above $80 \mathrm{~nm}$, which indicates a possible increase in diffuse scattering as the wire diameter approaches submicron size.

Further investigations are currently under way to gain a better understanding of the magneto-optical properties of the nanowire arrays.

We acknowledge gratefully the support by EPSRC and an ADF grant from the University of Salford. We wish to thank Prof. M. E. Pemble for providing chemistry laboratory space for the preparation of the samples, Dr. N. M. Boag for making available a UV-Vis spectrometer, Mr. M. Almasikashi for assistance, and Prof. P. J. Grundy for his interest and encouragement. One of the authors (Y.P.) would like to acknowledge an ORS award and a studentship from the University of Salford.

${ }^{1}$ T. Schmitte, T. Schemberg, K. Westerholt, H. Zabel, K. Schadler, and U. Kunze, J. Appl. Phys. 87, 5630 (2000).

${ }^{2}$ P. Vavassori, Appl. Phys. Lett. 77, 1605 (2000).

${ }^{3}$ M. T. Johnson, R. Coehoorn, J. J. de Vries, N. W. E. McGee, J. aan de Stregge, and P. J. H. Bloemen, Phys. Rev. Lett. 69, 969 (1992).

${ }^{4}$ M. J. Zheng, L. D. Zhang, G. H. Li, X. Y. Zhang, and X. F. Wang, Appl. Phys. Lett. 79, 839 (2001).

${ }^{5}$ M. El-Kouedi and C. A. Foss, Jr., J. Phys. Chem. B 104, 4031 (2000).

${ }^{6}$ A. Kirliyuk, Th. Rasing, B. Doudin, and J.-Ph. Ansermet, J. Appl. Phys. 81, 4723 (1997).

${ }^{7}$ S. Blom, L. Y. Gorelik, M. Jonson, R. I. Shekhter, A. G. Scherbakov, E. N. Bogachek, and Uzi Landman, Phys. Rev. B 58, 16305 (1998).

${ }^{8}$ J. C. Kemp, Polarized Light and its Interaction with Modulating Device-A Methodology Review (Hinds International, Inc., 1987).

${ }^{9}$ M. Born and E. Wolf, Principles of Optics, Sixth (corrected) ed. (Pergamon, New York, 1987), pp. 554-555.

${ }^{10}$ Y. Peng, H. L. Zhang, S. L. Pan, and H. L. Li, J. Appl. Phys. 87, 7405 (2000).

${ }^{11}$ P. N. Argyres, Phys. Rev. 97, 334 (1955).

${ }^{12}$ K. E. Gonsalves, G. Carlson, M. Benaissa, M. Jose-Yacaman, D. Y. Kim, and J. Kumar, J. Mater. Chem. 7, 703 (1997).

${ }^{13}$ R. B. Wagreich and C. C. Davis, IEEE Trans. Magn. 33, 2356 (1997).

${ }^{14}$ D. Almawlawi, N. Coombs, and M. Moskovits, J. Appl. Phys. 70, 4421 (1991).

${ }^{15}$ C. K. Preston and M. Moskovits, J. Phys. Chem. 97, 8495 (1993).
to AIP license or copyright, see http://ojps.aip.org/aplo/aplcr.js 